\title{
Energy-Efficient Location Estimation Using Variable Range Beacons in Wireless Sensor Networks
}

\author{
Muhammad Farooq-i-Azam*‡, Qiang Ni*, Ejaz A. Ansari ${ }^{\ddagger}$ and Haris Pervaiz* \\ ${ }^{*}$ School of Computing and Communications, Lancaster University, UK. \\ ${ }_{\ddagger}^{\ddagger}$ Department of Electrical Engineering, COMSATS IIT, Lahore, Pakistan. \\ Email: \{m.farooq-i-azam, q.ni, h.pervaiz\}@lancaster.ac.uk, \{fazam, dransari\}@ciitlahore.edu.pk
}

\begin{abstract}
In a randomly deployed wireless sensor network, sensor nodes must determine their own geographic position coordinates so that the collected data can be ascribed to the location from where it was gathered. We propose a localization algorithm which uses variable range beacon signals generated by varying the transmission power of beacon nodes. The algorithm does not use any additional hardware resources for ranging and estimates position using only radio connectivity by passively listening to the beacon signals. The algorithm is distributed, so each sensor node determines its own position and communication overhead is avoided. As the beacon nodes do not always transmit at maximum power and no transmission power is used by unknown sensor nodes for localization, the algorithm is also energy efficient. It also provides control over localization granularity. Simulation results show that the algorithm provides good accuracy under varying radio conditions.
\end{abstract}

Keywords-localization; positioning; algorithm; wireless sensor networks; location estimation; position estimation; range free; multilateration; energy efficiency.

\section{INTRODUCTION}

In many applications of wireless sensor networks, such as target tracking, habitat monitoring and area surveillance [1], [2], the sensor nodes are deployed randomly e.g. dropped from an airplane. In such cases, the unknown sensor nodes must determine their geographic position coordinates usually with the assistance of a few beacon nodes which know their positions a priori either because these are placed at pre-determined locations or are equipped with location finding hardware, such as, GPS. Due to energy and size limitations, all unknown sensor nodes cannot be equipped with such extra piece of hardware.

A number of techniques have been used to solve the problem of localization in wireless sensor networks. Their details are available in various literature surveys [3], [4]. In [5], an unknown sensor node determines its connectivity with neighbor beacon nodes and estimates its position at their centroid. Authors of [6] analyze the quantization of received signal strength (RSS) measurements for the purpose of location estimation. Localization technique in [7] uses RSS measurements to constrain the region of estimated location. In an iterative process, this estimate is propagated to help neighbor nodes improve their position estimates. Another algorithm described in [8] uses propagation of RSS information of beacon nodes to help unknown nodes localize.
In [9], an unknown node determines a region around itself constrained by points of intersection of beacon signals and then estimates its position at the center of the constrained region. The localization scheme in [10] builds a tripodal anchor structure and uses grid scan to localize unknown nodes.

In this paper, we present a localization algorithm which is distributed so that each unknown node can localize itself passively by just listening to beacon nodes. The algorithm does not require any extra piece of hardware to estimate range and position. Based on the fact that the algorithm sends out a ripple of beacon signals, we call it ripple localization algorithm (RLA) for convenience of reference. We also show quantitatively that the algorithm is energy efficient compared to localization techniques which transmit beacon signals at fixed radio range. Approximately $92 \%$ of the upper limit of energy efficiency can be attained by using 10 quantization levels of transmission power.

This paper has three major contributions. First, we propose a novel, distributed and energy-efficient localization algorithm, which gives a good localization accuracy. Second, we give a quantitative analysis of energy efficiency of the proposed algorithm. Third, the algorithm is simulated using practical irregular radio conditions and is compared with two other localization algorithms.

The rest of this paper is organized as follows. In section II, we describe the ripple localization algorithm and analyze its energy efficiency. We evaluate performance and analyze simulation results in section III and conclude with section IV.

\section{Ripple Localization Algorithm}

In this section, we start with assumptions of the sensor field and then describe the ripple localization algorithm. It consists of two parts - one part is executed by beacon nodes and the other by the unknown nodes. We also give a quantitative analysis of the energy efficiency of both parts of the algorithm.

\section{A. Sensor Field}

We consider an outdoor wireless sensor network in a two dimensional unobstructed sensor field with finite geographic boundaries in which the sensor and beacon nodes are deployed randomly. Radio range of unknown nodes is longer compared to their sensing range. Communication range of beacon nodes is longer than that of unknown sensor nodes so that beacon 
signals reach a large number of unknown sensor nodes at greater distances. We assume that all nodes are equipped with omnidirectional antennas, designed for sensor networks such as one described in [11], so that nodes communicate equally in all directions. We also assume that orthogonality of beacon signals is handled by a medium access control protocol. To discuss and explain the algorithm, we assume a perfectly circular radio range. However, for performance evaluation and simulation, we use a more practical irregular radio model [12] as shown in Fig. 1. Degree of irregularity (DOI) is used to denote the extent of irregularity in radio pattern and is defined as the maximum radio range variation per unit degree change in the direction of propagation.

\section{B. Algorithm for Beacon Nodes}

In ripple localization algorithm, a beacon node transmits beacon signals at different power levels corresponding to different transmission radii so that these radii fall into certain pre-determined quantized intervals ${ }^{1}$. The beacon nodes are tested and calibrated so that transmission radii corresponding to different power levels are recorded for embedding in beacon messages. Hence, unknown nodes receive more information each time they receive a beacon message. The unknown nodes use this information to achieve better accuracy in location estimation.

Transmission of successive beacon signals with different transmission power and hence different transmission distance is shown in Fig. 2. This is analogous to a ripple in water. It emanates from the center and travels outwards. In the same manner, each beacon node generates a ripple of beacon signals. A beacon node sends its first beacon signal with some set minimum transmission power. For each successive beacon signal, it increases the transmission power such that transmission radius of the beacon signal is longer by a step $d_{r}$ from the previous beacon signal. Beacon node increments transmission power with each successive beacon signal until maximum transmission power is reached, at which point, the beacon node resets and starts this process all over again. A typical beacon message is shown in Fig. 3. In this beacon message, $t_{0}$ is the time stamp, $\left(X_{b}, Y_{b}\right)$ are the position coordinates of the beacon node, $P_{t i}$ is the transmission

\footnotetext{
${ }^{1}$ Many sensor node platforms allow the transmission power to be set dynamically. For example, when using CC2420, an IEEE-802.15.4 compliant RF transceiver, transmission power for each packet can be set using CC2420PacketC.setPower() command under TinyOS.
}

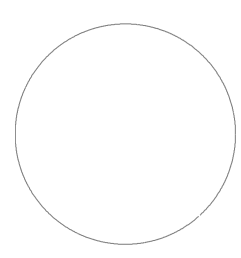

(a) $\mathrm{DOI}=0$

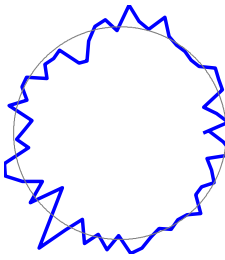

(b) $\mathrm{DOI}=0.1$

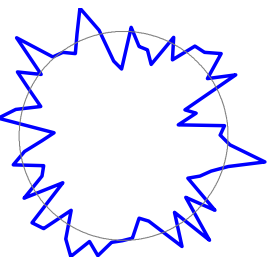

(c) $\mathrm{DOI}=0.2$
Fig. 1. Irregular radio pattern and degree of irregularity. power used, $R_{i}$ is the corresponding radio range, $d_{r}$ is the beacon signal step, $R_{\min }$ is the minimum transmission radius corresponding to the minimum transmission power, $P_{\min }$ and $R_{\max }$ is the maximum transmission radius corresponding to the maximum transmission power, $P_{\max }$.

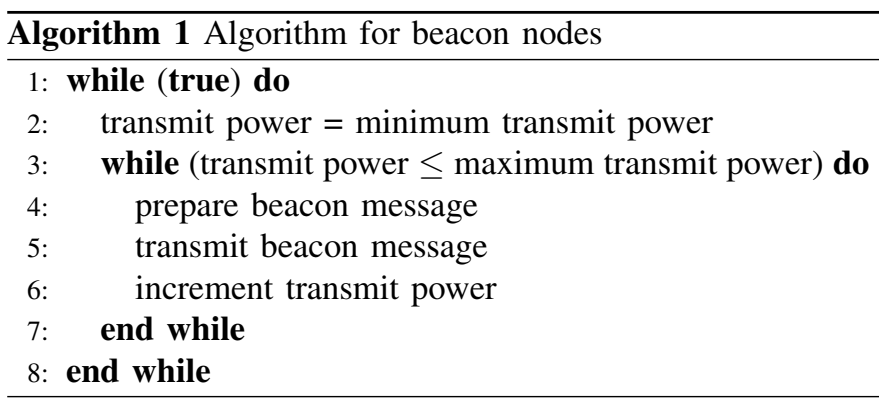

\section{Algorithm for Unknown Nodes}

Multiple unknown nodes lying within the communication range of beacon node receive its beacon signals as is shown in Fig. 2. By extracting information from all the beacon messages that an unknown node receives from a particular beacon node, it can determine the radii of the inner and outer circles of the annular ring around the beacon node in which it lies. For example, the first beacon signal that unknown node $U_{1}$ receives is beacon signal number 4 . Therefore, it can ascertain that outer radius of the annular ring in which it lies is the same as that of beacon signal 4 . Note that, of all the beacon messages that unknown node $U_{1}$ receives from that particular beacon node, first beacon signal has the smallest radius. Knowing the beacon signal step $d_{r}$ from the beacon message and by subtracting it from the radius of the outer circle, it can also determine the radius of the inner circle. Next, it estimates its distance from the beacon node by calculating

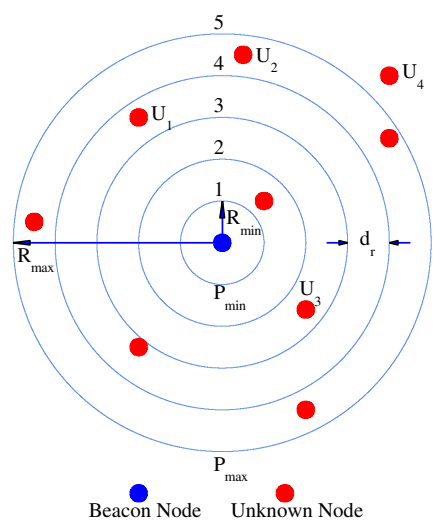

Fig. 2. A ripple of beacon signals.

\begin{tabular}{|l|l|l|l|l|l|l|l|}
\hline ID & $\mathrm{t}_{0}$ & $\left(\mathrm{X}_{\mathrm{b}}, \mathrm{Y}_{\mathrm{b}}\right)$ & $\mathrm{P}_{\mathrm{ti}}$ & $\mathrm{R}_{\mathrm{i}}$ & $\mathrm{d}_{\mathrm{r}}$ & $\mathrm{R}_{\min }$ & $\mathrm{R}_{\max }$ \\
\hline
\end{tabular}

Fig. 3. A typical beacon message. 
average of the radii of the inner and outer circles around the beacon node. In a similar manner, the unknown sensor node calculates its distance from each of its neighbor beacon nodes. Knowing geographic position coordinates of three or more neighbor beacon nodes and distances from them, the unknown node then constructs and solves a set of familiar multilateration equations of the following form to estimate its own position.

$$
\mathbf{z}=\mathbf{A}^{+} \mathbf{R}
$$

where $\mathbf{z}$ is a two-element column matrix comprising of estimated position coordinates $(x, y)$ of the unknown sensor node, $\mathbf{A}^{+}$is pseudoinverse of matrix $\mathbf{A}$ which is calculated using the known position coordinates of neighbor beacon nodes and matrix $\mathbf{R}$ is calculated using known position coordinates and estimated distances from neighbor beacon nodes. Details are available in literature.

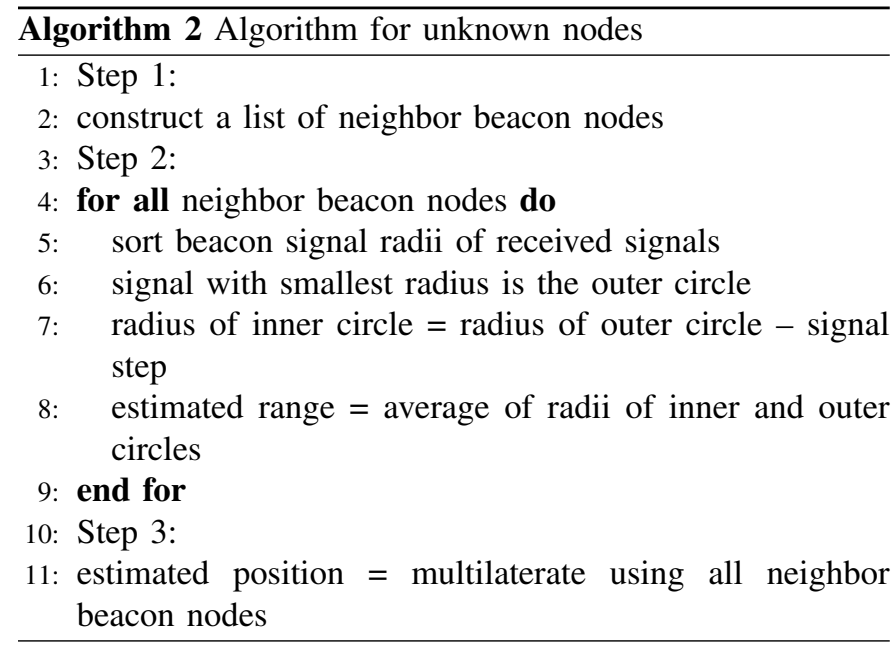

\section{Energy Efficiency}

We assume that relationship between transmitted power $P_{t}$ and received power $P_{r}$ between two nodes in the sensor field is governed by the following path loss model:

$$
P_{r}=P_{t} G_{t} G_{r}\left(\frac{\lambda}{4 \pi}\right)^{2}\left(\frac{1}{d}\right)^{\alpha}
$$

where $G_{t}$ and $G_{r}$ are gains of transmitter and receiver antennas respectively, $\lambda$ is the wavelength of radio waves, $d$ is the distance between transmitter and receiver antennas and $\alpha$ is the path loss exponent. Equation (2) can be rearranged as under:

$$
\frac{P_{t}}{P_{r}}=\frac{1}{G_{t} G_{r}}\left(\frac{4 \pi}{\lambda}\right)^{2} d^{\alpha}
$$

For a given pair of transmitter and receiver nodes in a sensor network, $G_{t}, G_{r}$ and $\lambda$ are constant. Therefore, (3) can be written as:

$$
\frac{P_{t}}{P_{r}}=K d^{\alpha}
$$

where

$$
K=\frac{1}{G_{t} G_{r}}\left(\frac{4 \pi}{\lambda}\right)^{2}
$$

Let us now successively increment the transmitted power from its minimum value $P_{\min }$ to maximum value $P_{\max }$ corresponding to beacon signal minimum radio range $R_{\text {min }}$ and beacon signal maximum radio range $R_{\max }$ respectively so as to generate a ripple of beacon signals as shown in Fig. 2. The power is increased such that with each increment of power, increase in beacon signal radio range remains the same i.e. difference between radii of two consecutive beacon signals remains constant. We call this distance as beacon signal step and denote it by $d_{r}$. Furthermore, let us also assume that beacon signal minimum radio range $R_{\min }$ is equal to beacon signal step $d_{r}$ for simplicity. Let the transmitted power corresponding to a beacon signal $i$ be denoted by $P_{t i}$ and radio range of the corresponding beacon signal be $R_{i}$. As the difference between the radii of two consecutive beacon signals $d_{r}$ is constant, therefore

$$
R_{i}=i \times d_{r}
$$

If the total number of beacon signals in the ripple generated by the beacon node is $n$, then

$$
R_{\max }=n \times d_{r}
$$

According to (4), transmitted power $P_{t i}$ for a beacon signal $i$ is given as:

$$
\frac{P_{t i}}{P_{r}}=K R_{i}^{\alpha}
$$

Similarly, maximum transmitted power $P_{\max }$ is given by

$$
\frac{P_{\max }}{P_{r}}=K R_{\max }^{\alpha}
$$

Received power $P_{r}$ is the same in (8) and (9). Substituting (6) in (8) and (7) in (9), we get:

$$
\begin{aligned}
\frac{P_{t i}}{P_{r}} & =K\left(i d_{r}\right)^{\alpha} \\
\frac{P_{\text {max }}}{P_{r}} & =K\left(n d_{r}\right)^{\alpha}
\end{aligned}
$$

Dividing (10) by (11), we get:

$$
P_{t i}=\left(\frac{i}{n}\right)^{\alpha} P_{\max }
$$

To get upper bound on the energy saved, we use $\alpha=2$. Therefore, total power $P_{T}$ transmitted by a beacon node for sending a ripple of $n$ beacon signals is given by:

$$
P_{T}=\frac{P_{\max }}{n^{2}} \sum_{i=1}^{n} i^{2}
$$

Summation term on the right is the sum of squares of first $n$ natural numbers, which is given by:

$$
\sum_{i=1}^{n} i^{2}=\frac{n(n+1)(2 n+1)}{6}
$$

Substituting this in (13), we get

$$
P_{T}=\frac{(n+1)(2 n+1)}{6 n} P_{\max }
$$


If a beacon node transmits 5 beacon messages, all at maximum power, transmit power used is $5 P_{\max }$. However, if a ripple of 5 beacon messages is transmitted by varying the transmit power, so that $n=5$, the total transmitted power, as calculated using (15) is $2.2 P_{\max }$, which is less than half of the power required to transmit usual beacon messages at maximum power. Power ${ }^{2}$ saved is $5 P_{\max }-2.2 P_{\max }=2.8 P_{\max }$ and energy efficiency of $100 \times 2.8 / 5=56 \%$ is achieved. In general, transmit power saved $P_{S}$ in transmitting a ripple of $n$ beacon signals is given by:

$$
P_{S}=n P_{\max }-\frac{(n+1)(2 n+1)}{6 n} P_{\max }
$$

This can be simplified to arrive at the following result:

$$
P_{S}=\frac{(4 n+1)(n-1)}{6 n} P_{\max }
$$

This gives us the energy saved when a ripple of beacon messages is sent instead of transmitting beacon signals at fixed radio range. If $n$ beacon messages are transmitted at fixed power, the transmitted power used is $n P_{\max }$. Percentage of power saved or energy efficiency $\eta_{P}$ achieved is given by:

$$
\eta_{P}=\frac{P_{S}}{n P_{\max }} \times 100=\frac{(4 n+1)(n-1)}{6 n^{2}} \times 100
$$

Note that for $n=1$, i.e. beacon messages with only one power level, (17) and (18) result in zero implying that no energy is saved. For $n=5, \eta_{P}$ is $56 \%$ which is the same as calculated earlier using (15). A plot of (18) for the interval $0 \leq n \leq 10$ is shown in Fig. 4. As can be seen, greater the number of beacon signals $n$, greater is the energy saved. In the limit, when a beacon node transmits an infinite number of beacon signals, maximum energy efficiency $\eta_{P \max }$ is achieved and is given by:

$$
\eta_{P \max }=\lim _{n \rightarrow \infty} \frac{\left(4+\frac{1}{n}\right)\left(1-\frac{1}{n}\right)}{6} \times 100=66.67 \%
$$

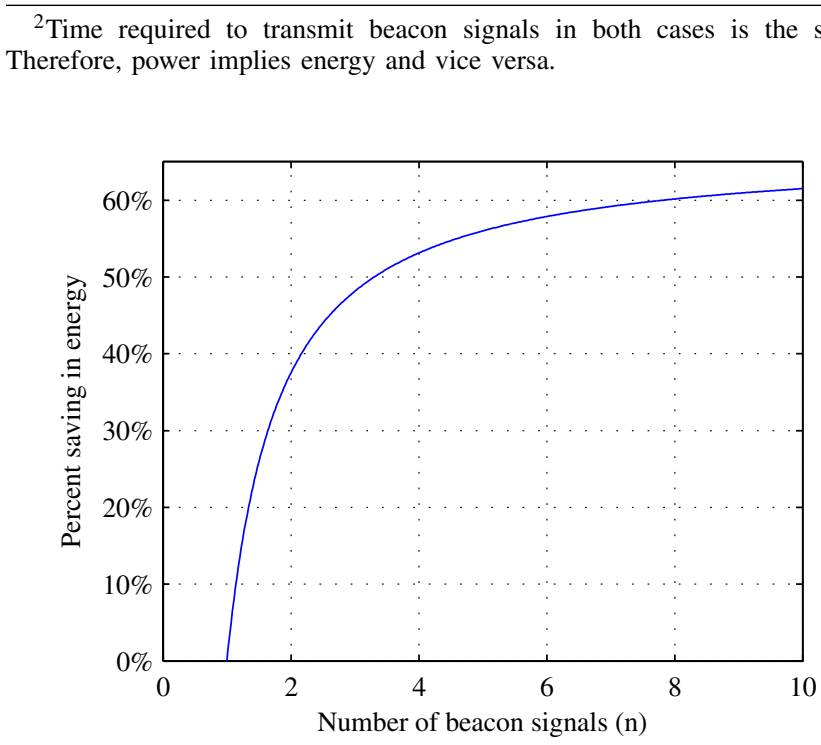

Fig. 4. Energy saving with increase in beacon signals in a ripple.
This shows that upper bound on the energy saved by a beacon node is $66.67 \%$ when the number of beacon signals in a ripple approaches $\infty$.

Using (18), we calculate that for $60 \%$ and $65 \%$ energy saving, the number of beacon signals in a ripple is approximately 8 and 30 respectively. For $n=10$ in a ripple, we get $61.50 \%$ energy saving i.e. we attain approximately $92 \%$ of the upper limit of energy efficiency.

The proposed algorithm does not require unknown node to transmit anything and it estimates the position passively by merely receiving and processing information from beacon nodes. Therefore, unknown nodes utilize zero transmission energy for the purpose of localization. We assert that the only energy an unknown node expends for localization is the processing energy. It is further added that we are concerned only with energy used by the localization algorithm and not the overall energy used by a sensor node for various other tasks.

\section{Simulation AND Results}

To evaluate performance of the algorithm, it is simulated using MATLAB. A square sensor field of size $100 \mathrm{~m} \times 100 \mathrm{~m}$ with 100 randomly deployed sensor nodes is used for the simulation experiments. Beacon nodes have a minimum $10 \mathrm{~m}$ and maximum $100 \mathrm{~m}$ transmission radius. A practical irregular radio model as depicted in Fig. 1 is used for performance evaluation. DOI, number of beacon nodes and number of beacon signals in a ripple are varied in a number of simulation tests and positions and localization errors are recorded. Localization error is the distance between actual and estimated positions normalized to sensor node radio range, $R_{s}$. All simulation results and data are available from the authors upon request.

\section{A. Number of Beacon Signals in a Ripple}

We vary the number of waves in the ripple i.e. number of beacon signals from 1 to 10 and record localization error. The number of beacon nodes used is $20 \%$. Results are plotted in Fig. 5. As more and more beacon signals are added in the ripple, the distance between the inner and outer radii of annular rings becomes smaller thereby resulting in a smaller localization error. Under adverse radio conditions with $\mathrm{DOI}=0.2$, mean error is below $0.75 R_{s}$ when $n=5$, and approximately $0.5 R_{s}$ when $n=10$. Therefore, depending upon application of deployed sensor network, localization granularity can be controlled by varying the number of beacon signals in a ripple. Higher the number of beacon signals $n$, finer is the location granularity.

\section{B. Number of Beacon Nodes}

Results are plotted in Fig. 6 as the number of beacon nodes in the sensor field is increased from $3 \%$ to $30 \%$ using $n=10$. Localization error is approximately $0.75 R_{s}$ with $\mathrm{DOI}=0.2$ and is $0.50 R_{s}$ with DOI $=0.1$ as the number of beacon nodes is increased to $10 \%$. If number of beacon nodes is increased beyond $15 \%$, only marginal gain in localization accuracy is achieved. 


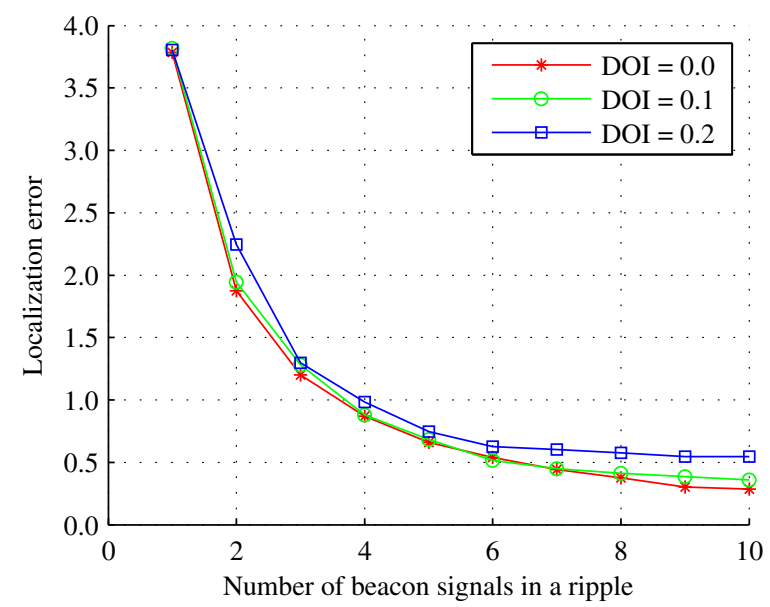

Fig. 5. Effect of number of beacon signals in a ripple on localization error.

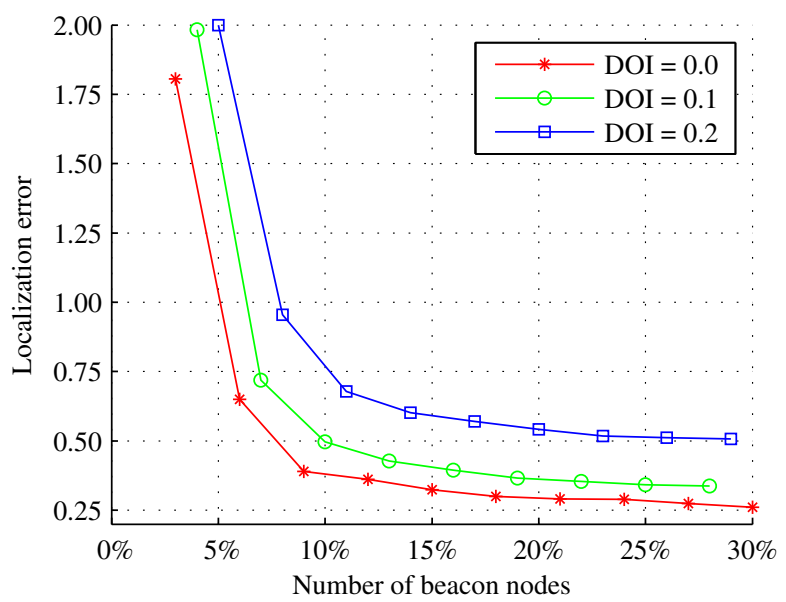

Fig. 6. Localization error with change in number of beacon nodes.

\section{Error Distribution}

Plot in Fig. 7 is a CDF of the localization error using $10 \%$ beacon nodes and $n=10$. Approximately $95 \%$ nodes have error below $0.45 R_{s}$ under ideal radio conditions. Under adverse radio conditions with DOI $=0.2$, approximately $85 \%$ nodes have localization error below $0.80 R_{s}$.

\section{Performance Comparison}

Performance of ripple localization algorithm (RLA) is compared with two other distributed algorithms - Centroid [5] and concentric anchor beacon (CAB) algorithm [9]. Under adverse radio conditions, with $\mathrm{DOI}=0.2$, localization error is recorded while number of beacon nodes in the sensor field is varied from $3 \%$ to $30 \%$. Results, plotted in Fig. 8, show that RLA performs better than both Centroid and CAB over the entire range of number of beacon nodes. At $10 \%$ beacon nodes, RLA has a localization error of approximately $0.75 R_{s}$ whereas it is approximately $3.75 R_{s}$ for Centroid and $1.45 R_{s}$ for $\mathrm{CAB}$. Addition of beacon nodes beyond $15 \%$ does not result in significant improvement in localization accuracy of

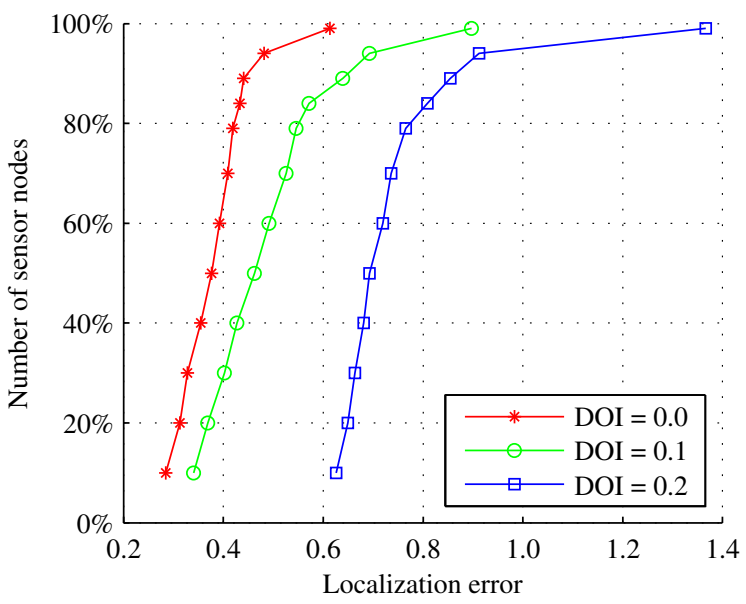

Fig. 7. Cumulative error distribution.

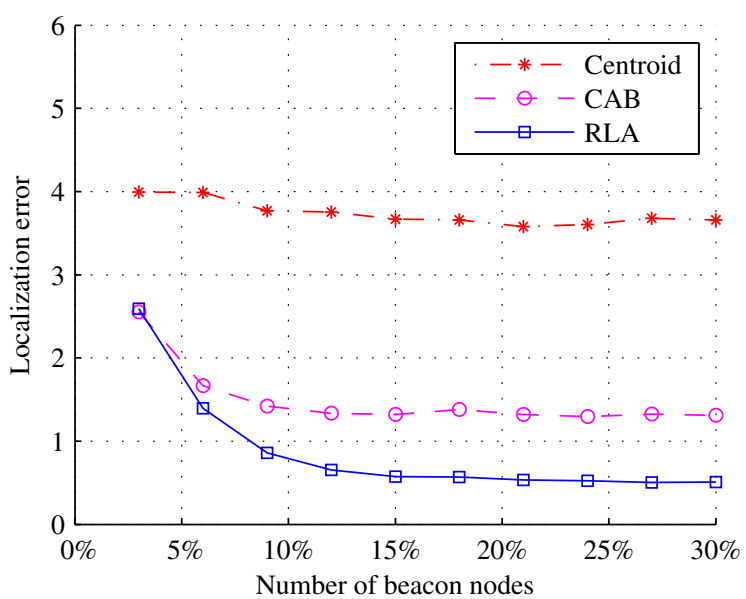

Fig. 8. Performance comparison of Centroid, CAB and RLA.

any of the three localization algorithms.

\section{CONCLUSION}

We have presented an energy efficient distributed localization algorithm for wireless sensor networks. Performance of the algorithm is evaluated using simulation and results show that the algorithm provides good localization accuracy even when using a small percentage of beacon nodes. The algorithm is able to estimate position without using any additional piece of hardware thereby saving cost, size and energy. The algorithm does not require unknown nodes to expend any transmission energy and they can localize passively using only processing energy by merely listening to the beacon messages. The algorithm saves $66.67 \%$ energy in transmission of beacon signals compared to the algorithms that transmit beacons at fixed maximum power. Approximately $92 \%$ of this energy efficiency can be achieved using 10 discrete power levels. It also provides control over localization granularity. 


\section{REFERENCES}

[1] M. P. Michaelides, C. Laoudias, and C. G. Panayiotou, "Fault tolerant localization and tracking of multiple sources in WSNs using binary data," IEEE Transactions on Mobile Computing, vol. 13, no. 6, pp. 1213 - 1227, June 2014.

[2] L. Parra, S. Sendra, J. Lloret, and J. J. Rodrigues, "Low cost wireless sensor network for salinity monitoring in mangrove forests," in Proceedings of IEEE Sensors, November 2014, pp. 126 - 129.

[3] G. Han, H. Xu, T. Q. Duong, J. Jiang, and T. Hara, "Localization algorithms for wireless sensor networks: a survey," Telecommunication Systems, vol. 52, no. 4, pp. 2419 - 2436, April 2013.

[4] L. Cheng, C. Wu, Y. Zhang, H. Wu, M. Li, and C. Maple, "A survey of localization in wireless sensor networks," International Journal of Distributed Sensor Networks, November 2012.

[5] N. Bulusu, "Self-configuring localization systems," Ph.D. dissertation, University of California, Los Angeles, 2002.

[6] N. Patwari and A. O. Hero III, "Using proximity and quantizied RSS for sensor localization in wireless networks," in Proceedings of the 2nd ACM International Conference on Wireless Sensor Networks and Applications, 2003, pp. 20 - 29.

[7] M. L. Sichitiu, V. Ramadurai, and P. Peddabachagari, "Simple algorithm for outdoor localization of wireless sensor networks with inaccurate range measurements," in Proceedings of International Conference on Wireless Networks, Las Vegas, 2003, pp. 300 - 305
[8] C. Liu, K. Wu, and T. He, "Sensor localization with ring overlapping based on comparison of received signal strength indicator," in Proceedings of IEEE International Conference on Mobile Ad-hoc and Sensor Systems, October 2004, pp. 516 - 518.

[9] V. Vivekanandan and V. W. S. Wong, "Concentric anchor beacon localization algorithm for wireless sensor networks," IEEE Transactions on Vehicular Technology, vol. 56, no. 5, pp. 2733 - 2744, September 2007.

[10] H.-W. Ferng and I. Arwani, "Concentric distributed localization based on the tripodal anchor structure and grid scan for wireless sensor networks," Wireless Personal Communications, vol. 68, no. 4, pp. 1707 - 1729, February 2013.

[11] W. Hong and K. Sarabandi, "Design of low-profile omnidirectional antenna for ground sensor networks," in IEEE Antennas and Propagation Society International Symposium, June 2007, pp. 6007 - 6010

[12] T. He, C. Huang, B. M. Blum, J. A. Stankovic, and T. Abdelzaher, "Range-free localization schemes for large scale sensor networks," in Proceedings of the 9th Annual International Conference on Mobile Computing and Networking. ACM, 2003, pp. $81-95$. 